\title{
Multi-criteria Trajectory Planning for Hepatic Radiofrequency Ablation
}

\author{
Claire Baegert ${ }^{1,2}$, Caroline Villard ${ }^{2}$, Pascal Schreck ${ }^{2}$, and Luc Soler ${ }^{1}$ \\ ${ }^{1}$ IRCAD, 1 place de l'Hôpital, 67000 Strasbourg \\ ${ }^{2}$ LSIIT, Pôle API, Bd Sebastien Brand, 67412 Illkirch \\ \{baegert, villard, schreck\}@lsiit.u-strasbg.fr, \\ luc.soler@ircad.u-strasbg.fr
}

\begin{abstract}
In this paper, we propose a method based on multiple criteria to assist physicians in planning percutaneous RFA on liver. We explain how we extracted information from literature and interviews with radiologists, and formalized them into geometric constraints. We expose then our method to compute the most suitable needle insertion in two steps: computation of authorized insertion zones and multi-criteria optimization of the trajectory within this zones. We focus on the combination of the criteria to optimize and on the optimization step.
\end{abstract}

\section{Introduction}

New minimally invasive methods have recently been developed to treat patients with not resectable liver tumors. These methods achieve in situ destruction thanks to chemical agents or temperature. We focus on percutaneous radiofrequency ablation (RFA) that is one of the most used and effective methods, and involves only a short hospital stay and a reduced cost. Guided by CT or ultrasound images, the physician inserts a needle through the patient's skin. A radiofrequency alternating current flow is then delivered so that tissues close to the needle tip are heated and coagulate above $60^{\circ} \mathrm{C}$.

Experience of the operator highly affects the chances of a complete ablation and the risks of complications [1]. Our work is aiming at providing a planning software based on collected physicians expertises to recommend an optimal strategy for each operation. In the following, we first recall other works that have already been accomplished in the domain of minimally invasive surgery planning. Secondly, we expose how we have formalized some information from the expertise of radiologists to define criteria influencing the strategies. Then we explain our methods to merge and solve them in order to propose an adapted solution for each operation. Finally, we evaluate our results on virtual patients and we discuss the further developments.

\section{Related Works}

We wish our planning software to be used in the operating room, after the CT acquisition and before the intervention in order to work on accurate data. This gives about 30 minutes for both reconstruction and planning processes. The planning process consists of choosing the best strategy among a lot of candidate trajectories, taking into 
account the whole anatomy of the patient. That is why, for each considered trajectory, the corresponding necrosis zone (that we will call lesion in the rest of the paper) must be predicted as precisely and quickly as possible.

Several studies focused on automatically optimizing tools position in order to minimize damage to surrounding tissues. Altrogge et al. [2] proposed an optimization method based on the simulation of temperature within tissues. However the resulting trajectory does not take into account surrounding organs and no computation time is indicated. Butz et al. [3] focused on cryoablation. They proposed an optimization of probes position in a secure window provided by the physician. Other studies related to prostate cryosurgery also have to be mentioned [4] [5]. Both works combine planning with thermal exchange simulation, with the drawback of a long computation time. Moreover one of the models is restricted to $2 \mathrm{D}$ and the second requires manual interventions for optimization.

Interesting works have been performed on computer planning of robotically assisted minimally invasive surgery for heart interventions [6]. Like in RFA planning, the issue is to propose a strategy that respects several constraints and optimizes several criteria. However, optimization is performed by an exhaustive search within a very limited number of incision sites, that would not be possible in a reasonable time in our case, as we showed in [7], as the search domain is too wide. For this reason, we focused our studies on a multi-criteria optimization process.

\section{Characterization of the Constraints Governing RFA Planning}

In an earlier paper [8] we were taking into account one single constraint: the inclusion of the entire tumor while minimizing the amount of destroyed healthy tissues. However in practice several criteria are considered, some of them being disqualifying, others being to optimize. The rules that motivate the strategy for each operation are not clearly enunciated and may vary between specialists. However, the most essential of them appear recurrently. In this section we selected some of those recurrent criteria from medical literature, that were confirmed by our expert practitioners. Of course the weights of the criteria can be adjusted and extra criteria could be added (see section 3.3).

\subsection{Analysis of Medical Literature}

Although RFA is a recent technique, many medical studies that detail the different aspects of this operation have been published. We focus on liver tumors RFA, but our work could easily be adapted to other cancer location. We consider the percutaneous approach for which the preoperative planning takes an important place because of the limited visibility during the intervention.

RFA is generally conceivable for non-resectable small tumors (smaller than $5 \mathrm{~cm}$ ). The ablation of bigger tumors is possible but often requires multiple needle insertions that raise the risk of misplacement and incomplete tumor destruction [9]. The RF-lesion has to include a $0.5-1 \mathrm{~cm}$ margin around the tumor. Lesion shape and size vary according to specific material used [10]. The theoretical shape of the lesion is a spheroid, with different small/big axis ratio according to the needle model. In practice shape is influenced by the cooling effect of large vessels in the neighbourhood [11]. 
The operation is successful if no recurrent tumor is noticed at the original site during the follow-up. Rates of local recurrence vary between studies depending on different parameters [12], but tumor's size, location and physician's experience highly influence chances of success. Different kinds of complication can occur [13]. Patient's organs, vessels, or bile ducts can be damaged during needle placement or thermal ablation. Cancerous cells may adhere to the needle during its removal and result in the development of a new tumor along the needle path. Remaining functional liver may have been overestimated. The needle trajectory must be chosen in order to minimize these risks.

\subsection{Selected Constraints}

The constraints cited by specialists can be classified into 2 categories: strict constraints and soft constraints. A needle trajectory (considered in a first approach as rectilinear) has to fulfill all strict constraints to belong to the solution space. Among all solutions, the proposed trajectory has to satisfy at best the soft constraints. We selected the following four strict constraints that have to be fulfilled:

- The trajectory must not cross any vital organ, bone or major blood vessel,

- The insertion depth must be below needle size,

- The insertion angle in the liver must not be to tangent to liver surface in order to prevent risks of gliding on the surface during insertion,

- The trajectory must include a safe portion of the liver in order to enable cauterization of the path.

Among all solutions, the final strategy has to optimize soft constraints. We selected the three following ones:

- Volume of healthy tissues ablated: the needle should be placed so that the shape of the RF-lesion is as close as possible to target volume: remaining hepatic reserve is maximized and ablation can be done in a minimal number of needle insertions.

- Distance to vital organs: a trajectory that is very close to vital organs should often be penalized because in practice a little deviation from the planned trajectory is unavoidable. It is important to minimize risks of fatal injury.

- Insertion depth: short trajectories are often privileged because long trajectories increase risks of imprecision.

These categories of contraints are different by nature: soft constraints are continuous, whereas strict constraints are boolean. Strict contraints are combined using a simple intersection of their solution spaces (see section 4.1). For soft constraints, we chose a global approach that merges them into a unique function to optimize. In the following section we present this approach and discuss about other combination possibilities.

\subsection{Determination of the Global Minimization Function}

First, let us describe more precisely our optimization problem. The following functions express formally the different constraints we want to optimize: 
- lesion_volume $: \mathbb{R}^{5} \rightarrow \mathbb{R}_{+}$, denoting the volume of the minimal lesion including the tumor and margin, according to the 5 degrees of freedom of the needle ( 3 for the needle tip position and 2 for the angles),

- depth $: \mathbb{R}^{5} \rightarrow \mathbb{R}_{+}$, denoting the depth of insertion (distance between insertion point and target point),

- distance : $\mathbb{R}^{5} \times O \rightarrow \mathbb{R}_{+}$, denoting the minimal distance from the needle to an organ belonging to the set $\mathrm{O}$ of surrounding organs.

The rough combination of these functions would be meaningless, since they do not have the same order of magnitude. We then consider pseudo-normalizations performed specifically for each function in adequacy with its semantic before combining them.

For function lesion_volume, we define function $f_{v}$ by the formula

$$
f_{v}(X)=\frac{\text { lesion_volume }(X)-\text { min }_{x \in D}(\text { lesion_volume }(x))}{P . \min _{x \in D}(\text { lesion_volume }(x))}
$$

where $X$ represents any needle placement, $D$ the set of eligible placements that correspond to an appropriate access to the tumor. $P$ is a critic proportion of volume above which the volume loss is considered too important (experimental value: 60\%).

Function depth is also linear, because we think that the penalty increases linearly according to the depth of insertion as well. We consider function $f_{d}$ defined by

$$
f_{d}(X)=\frac{\operatorname{depth}(X)-\min _{x \in D}(\operatorname{depth}(x))}{\text { needle_length }-\min _{x \in D}(\operatorname{depth}(x))}
$$

For function distance, we want the measure of the risk to increase more quickly when the needle comes close to an organ. To this end, it is convenient to use a square root function. We also want to add the collision risks for all the organs, so we simply use a sum. Therefore, we obtain function $f_{r}$ defined by

$$
f_{r}(X)=\sum_{o \in O} \sqrt{\max \left(0, \frac{\text { dist_limit }_{o}-\text { distance }(X, o)}{\text { dist_limit }_{o}}\right)}
$$

where dist_limit $t_{o}$ represents a set of parameters representing a security distance that sould be observed for each organ. In order to eliminate the risk of a negative value inside the square root, we take the max between 0 and the value found. A negative value would occur if the needle is significantly far from the organ, so it is acceptable to consider in that case that the function is minimized and equal to zero.

We then define function $f_{\text {all }}$ that is a linear combination of the three others:

$$
f_{\text {all }}(X)=a_{v} \cdot f_{v}(X)+a_{r} \cdot f_{r}(X)+a_{d} \cdot f_{d}(X)
$$

with $a_{v}, a_{r}, a_{d} \in[0,1]$ and $a_{v}+a_{r}+a_{d}=1$. These three weights represent the importance of each criteria in the final decision. A linear combination has been chosen because it is a predictable and intuitive function, and provides weights to act on each constraint. It can be objected that the minimization of this function could result in a trajectory that satisfies badly one of the constraints. However if one soft constraint must be satisfied more than the others, the corresponding weight should be set in consequence 

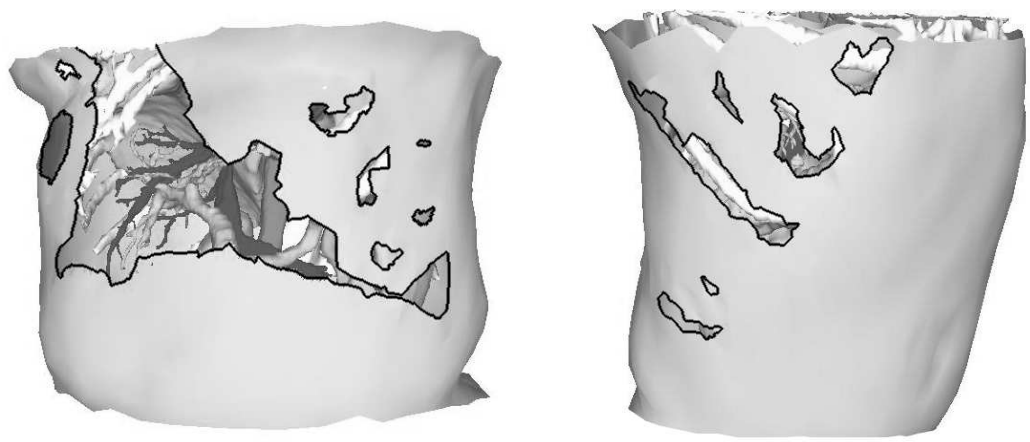

Fig. 1. Examples of insertion zones: Holes in the skin represent possible needle access to the tumor

or it should be included in strict constraints. Finally, the proposed trajectory respects the strict constraints and minimizes the final function $f_{\text {all }}$. In the following section, we present the method we developed to compute this trajectory.

\section{Determination of the Needle Insertion Strategy}

\subsection{Determination of Possible Solutions}

First of all, before choosing an optimal path, it is important to determine the set of all possible trajectories with precision. We developed a method that automatically computes the possible insertion zones on the skin and that has been the subject of an earlier publication [14]. A needle insertion in the resulting zone guarantees that the strict constraints presented earlier are satisfied. Then a trajectory is considered as eligible and belongs to $D$ only if it crosses the insertion zone (see examples of zones on Fig. (1).

\subsection{Optimization Phase}

Like in most of optimization problems, we face a large number of possibilities that cannot be entirely studied in a reasonable computation time. Moreover most of existing optimization methods do not completely avoid the problem of local minima. We developed a method in two steps. The initialization step finds, after a rough discretization of the angular space, one or more trajectories that seem close to the minimal. Then these trajectories are used as starting points for a local minimization and resulting values are compared to select the best choice. In a previous paper [7], we showed that this two-step method was fast and efficient on local minima, by comparing with the exhaustive one, with one single criterion.

The initialization step of multi-criteria optimization consists in discretizing the angular parameters space, by evaluating $f_{\text {all }}$ only for trajectories with a chosen step of $6^{\circ}$ with a fixed needle tip position. In this discretization we select trajectories resulting in a nearly minimal evaluation $\left.\left(f_{\text {all }}(t)=\min _{u \in \text { zone }}\left(f_{\text {all }}(u)\right)+\epsilon\right)\right)$. From each selected 
trajectory, we perform a local optimization thanks to downhill simplex method that has proven to converge quickly and to provide precise results in our earlier works.

\subsection{Results}

Our method has been tested on 16 tumors in 7 virtual reconstructions of real patient cases and results are shown on Tab.11 For all these patients, the total optimization phase (initialization + local optimization for each initial trajectory) took around 30 seconds, with a pentium $43.2 \mathrm{GHz}, 2 \mathrm{Go}$ RAM with a GeForce $7800 \mathrm{GT}$. Most of the time, one or two trajectories are selected by the initialization step. The trajectory proposed for each case is quite satisfying with respect to the three criteria. If we compare the volume of the lesion of the proposed trajectory with the minimal lesion possible volume in the insertion zone, we notice that the volume loss is low (average $4,4 \%$ more than min. vol.). Insertion depth of the optimal trajectories are around $5 \mathrm{~cm}$ that should facilitate a precise placement of the needle during the operation. In most of the case no vital structures are approached within $1 \mathrm{~cm}(0.5 \mathrm{~cm}$ for vessels $)$ of the needle trajectory. In case 13 vena cava is $5 \mathrm{~mm}$ and in case 15 lung is $6.6 \mathrm{~mm}$ close to the final trajectory. In both cases, proximity is unavoidable because of the location of the tumors. In all cases it is possible to determine quickly what are the best access points.

The results of the initialization can be visualized as color maps on the skin (see Fig 2). The software also provides the possibility to interfere in the proposed solution: the weights of each criteria (default: $\frac{1}{3}$ ) can be modified by the physician if he wishes to privilege one criteria. Once the optimization phase has been performed, a modification of the weights results in a real-time update of the initialization and of the color maps. An additional local optimization is sufficient to update the optimal trajectory.

Table 1. Evaluation of the optimal trajectory regarding the soft constraints

\begin{tabular}{|c|c|c|c|c|c|}
\hline case & $\begin{array}{c}\text { no. of } \\
\text { traj. }\end{array}$ & $\begin{array}{c}\text { tot. opt. } \\
\text { time }(\mathrm{s})\end{array}$ & $\begin{array}{c}\text { lesion vol. (min. } \\
\text { vol.) }(\mathrm{cm} 3)\end{array}$ & $\begin{array}{c}\text { close vital } \\
\text { structures }(\mathrm{mm})\end{array}$ & $\begin{array}{c}\text { insertion depth } \\
(\mathrm{cm})\end{array}$ \\
\hline 1 & 1 & 27 & $12.3 \mathbf{( 1 1 . 7 )}$ & none & 4.2 \\
\hline 2 & 2 & 24 & $3.3 \mathbf{( 3 . 3 )}$ & none & 4.9 \\
\hline 3 & 1 & 20 & $3.2 \mathbf{( 3 . 0})$ & none & 4.3 \\
\hline 4 & 1 & 18 & $2.5(\mathbf{2 . 5})$ & none & 5.9 \\
\hline 5 & 2 & 34 & $2.7(\mathbf{2 . 7})$ & none & $(5.4)$ \\
\hline 6 & 2 & 32 & $9.4(\mathbf{9 . 3})$ & none & 6.5 \\
\hline 7 & 3 & 32 & $10.1(\mathbf{9 . 6})$ & none & 5.0 \\
\hline 8 & 2 & 24 & $5.9(\mathbf{5 . 9})$ & none & 5.32 \\
\hline 9 & 1 & 17 & $6.6(\mathbf{6 . 0})$ & none & 4.2 \\
\hline 10 & 2 & 31 & $3.3 \mathbf{( 3 . 1}$ & none & 6.6 \\
\hline 11 & 1 & 24 & $4.8(\mathbf{4 . 6})$ & none & 5.3 \\
\hline 12 & 2 & 24 & $5.0(\mathbf{5 . 0})$ & none & 5.7 \\
\hline 13 & 2 & 22 & $3.9(\mathbf{3 . 4})$ & vena cava: 5.0 & 7.1 \\
\hline 14 & 3 & 29 & $3.1(\mathbf{2 . 8})$ & none & 8.7 \\
\hline 15 & 2 & 21 & $8.5(\mathbf{8 . 4})$ & right lung: 6.6 & 12.2 \\
\hline 16 & 1 & 17 & $5.7(\mathbf{5 . 4})$ & none & 6.3 \\
\hline
\end{tabular}




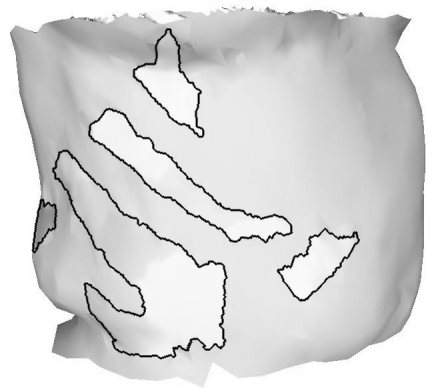

(a) Volume constraint

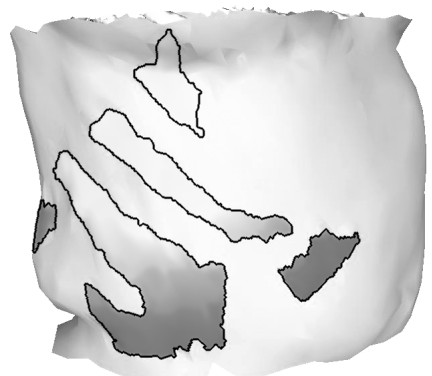

(c) Depth constraint

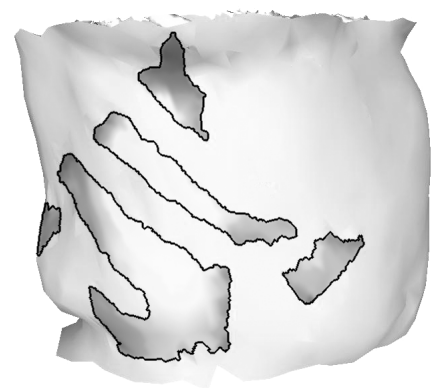

(b) Risk constraint

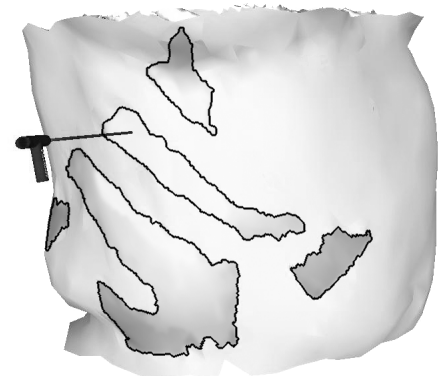

(d) Mix of the 3 weighted constraints

Fig. 2. Proposed trajectory and accuracy regarding the different constraints. Best locations are light-colored and worst locations are dark-colored.

\section{Conclusion and Future Works}

We proposed two kinds of geometric constraints to formalize medical expertise in planning RFA treatment. The resolution of strict constraints results in an insertion zone on the skin representing possible trajectories for the specific case. Among them a trajectory that satisfies at best the soft constraints has to be chosen. We proposed a minimization function that represents the different constraints affected by their respective weights. We solved the optimization problem in two steps: initialization thanks to a global study of the problem then local optimization from interesting needle positions. We showed that our method proposes a satisfying result regarding selected constraints in a few minutes.

Our results were shown to radiologists. For a few cases, we compared informally their estimated strategy with our computed trajectory. Although we did not perform numerical measurements, we noticed that we obtained close results, except once where we gave a better proposition according to the clinician. In addition to the theoretical validation exposed in this paper and the assessment by experts, we plan to implement a functionality to compare numerically our result with per-operative images. Further discussions with radiologists will also allow us to continue studying with more precision the implicit rules governing RFA, and even to make new constraints appear. 
RFA is a recent technique and corresponding rules and devices can evolve in the future. Moreover, the approach to each operation can vary between physicians. For these reasons, we want to have a flexible software that can adapt itself to changing constraints. Constraints definition is currently directly integrated in the software's code and cannot easily be modified. It could be worthwile to separate it from the software and to make it accessible for enhancements by expert users. We are thinking about using declarative modeling to achieve this. This way, our software could be easily adapted to other cancer location or to other kind of minimally invasive therapies.

\section{Acknowledgment}

We would like to thank Pr J. Marescaux, Region Alsace and research program IRMC for their financial support. We are also grateful to Pr Gangi, Pr Pereira, and Dr Buy for sharing their expertise.

\section{References}

1. Poon, R., Ng, K., Lam, C., Ai, V., Yuen, J., Fan, S., Wong, J.: Learning curve for radiofrequency ablation of liver tumors: Prospective analysis of initial 100 patients in a tertiary institution. Annals of Surgery 239, 441-449 (2004)

2. Altrogge, I., Kröger, T., Preusser, T., Büskens, C., Pereira, P., Schmidt, D., Weihusen, A., Peitgen, H.O.: Towards optimization of probe placement for radio-frequency ablation. In: Larsen, R., Nielsen, M., Sporring, J. (eds.) MICCAI 2006. LNCS, vol. 4190, pp. 486-493. Springer, Heidelberg (2006)

3. Butz, T., Warfield, S.K., Tuncali, K., Silverman, S.G., Van Sonnenberg, E., Jolesz, R., Kikinis, F.A.: Pre- and intra-operative planning and simulation of percutaneous tumor ablation. In: Delp, S.L., DiGoia, A.M., Jaramaz, B. (eds.) MICCAI 2000. LNCS, vol. 1935, pp. $317-$ 326. Springer, Heidelberg (2000)

4. Lung, D.C., Stahovich, T.F., Rabin, Y.: Computerized planning for multiprobe cryosurgery using a force-field analogy. Computer Methods in Biomechanics and Biomedical Engineering 7(2), 101-110 (2004)

5. Baissalov, R., Sandison, G.A., Donnelly, B.J., Saliken, J.C., McKinnon, J.G., Muldrew, K., Rewcastle, J.C.: A semi-empirical treatment planning model for optimization of multiprobe cryosurgery. Physics in Medicine and Biology 45, 1085-1098 (2000)

6. Adhami, L., Coste-Manière, E.: Optimal planning for minimally invasive surgical robots. IEEE Transactions on Robotics and Automation: Special Issue on Medical Robotics 19(5), 854-863 (2003)

7. Baegert, C., Villard, C., Schreck, P., Soler, L.: Trajectory optimization for the planning of percutaneous radiofrequeny ablation on hepatic tumors. Computer Aided Surgery (2007)

8. Villard, C., Baegert, C., Schreck, P., Soler, L., Gangi, A.: Optimal trajectories computation within regions of interest for hepatic rfa planning. In: Duncan, J.S., Gerig, G. (eds.) MICCAI 2005. LNCS, vol. 3750, pp. 49-56. Springer, Heidelberg (2005)

9. Curley, A.: Radiofrequency ablation of malignant liver tumors. Annals of Surgical Oncology 10(4), 338-347 (2003)

10. Mulier, S., Ni, Y., Miao, Y., Rosière, A., Khoury, A., Marchal, G., Michel, L.: Size and geometry of hepatic radiofrequency lesions. EJSO 29, 867-878 (2003) 
11. De Baere, T., Denys, A., Wood, B.J., Lassau, N., Kardache, M., Vilgrain, V., Menu, Y., Roche, A.: Radiofrequency liver ablation: experimental comparative study of water-cooled versus expandable systems. AJR 176(1), 187-192 (2001)

12. Mulier, S., Ni, Y., Jamart, J., Ruers, T., Marchal, G., Michel, L.: Local recurrence after hepatic radiofrequency coagulation. Annals of surgery 242, 158-171 (2005)

13. Rhim, H., Dodd, G.D., Chintapalli, K., Wood, B.J., Dupuy, D.E., Hvizda, J.L., Sewell, P.E., Goldberg, S.N.: Radiofrequency thermal ablation of abdominal tumors: Lessons learned from complications. Radiographics 24, 41-52 (2004)

14. Baegert, C., Villard, C., Schreck, P., Soler, L.: Precise determination of regions of interest for hepatic rfa planning. In: SPIE Medical Imaging (2007) 